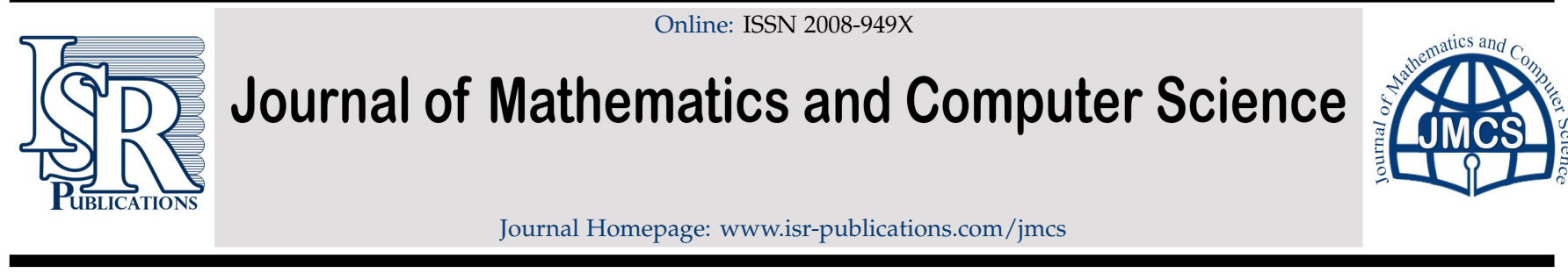

\title{
Cauchy problem for inhomogeneous fractional nonclassical diffusion equation on the sphere
}

\author{
Le Dinh Long \\ Division of Applied Mathematic, Thu Dau Mot University, Binh Duong province, Viet Nam.
}

\begin{abstract}
Pseudo-parabolic equation on spheres have many important applications in physical phenomena, oceanography and meteorology, geophysics. The main purpose of this paper is to prove the existence and unique solution of the nonlinear pseudoparabolic equation on the sphere. To do this, we used some analysis of Fourier series associated with several evaluations of the spherical harmonics function. Some of the upper and lower bounds of the Mittag-Lefler functions are also used. This result is one of the first studies of fractional nonclassical diffusion equation on the sphere.
\end{abstract}

Keywords: Fractional diffusion equation, Riemman-Liouville, regularity.

2020 MSC: 35R11, 35B65, 26A33.

(C)2022 All rights reserved.

\section{Introduction}

In geophysical phenomena, there will arise a number of problems related to the sphere. These problems are simulated by the partial differential equations on the sphere, and have many applications in physical geodesy, potential theory, oceanography and meteorology. The weather forecasting processes are also investigated and studied by mathematical models on the sphere. Therefore, we can say that the research on the sphere attracts quite a lot of mathematicians because of its applicability. Some mathematical analysis for parabolic equation on the sphere and numerical apprimation of it has been considered by many authors $[11,13,27]$. Cauchy problem for elliptic equations on the sphere has been studied in $[10,12]$. The Navier-Stokes equations on the 2D unit sphere has been investigated by recent paper [6]. Recently, Phuong and Luc [21] considered a pseudo-parabolic equation on spheres with classical derivative. We observe that the structure of the solutions of the differential equations on the sphere is very complex, so examining all kinds of partial derivative equations on the sphere requires advanced tools with new techniques. Motivated by this interesting result about pseudo-parabolic equation on the sphere with classical derivative, in this paper, we will study the problem of fractional nonclassical diffusion equation (or called pseudo-parabolic equation) on the sphere with fractional derivative.

According to the history of development in analytics, in the last few decades, fractional calculation has been a concept that has had a great influence on mathematics and the problems related to it often

Email address: ledinhlong@tdmu.edu.vn (Le Dinh Long)

doi: $10.22436 /$ jmcs.025.04.01

Received: 2021-01-15 Revised: 2021-03-10 Accepted: 2021-04-20 
have applications in modeling visualize real world problems. Fractional analysis has many applications in mechanics, physics and engineering science, etc. We would like to share many published works on these issues such as [1-3, 5, 9, 25], Jafari and his group [7, 8, 14, 19].

The main purpose of our present paper is to investigate the problem of pseudo-parabolic equation on the sphere with fractional derivative. Indeed, we introduce the pseudo-parabolic equation defined on the unit sphere $S^{2} \subset R^{3}$ as follows

$$
\frac{\partial^{\beta}}{\partial t^{\beta}} u(x, t)-\Delta^{*} u(x, t)-a \frac{\partial^{\beta}}{\partial t^{\beta}} \Delta^{*} u(x, t)=\psi(t) H(x, t), \quad(x, t) \in S^{2} \times[0, T]
$$

with the initial Cauchy condition

$$
u(x, 0)=\varphi(x)
$$

or the terminal Cauchy condition

$$
u(x, T)=\theta(x)
$$

where $\frac{\partial^{\alpha}}{\partial t^{\alpha}}$ is called the Riemann-Liouville fractional derivative of order $\alpha, 0<\alpha \leqslant 1$. It is defined by

$$
\frac{\partial^{\alpha} v(t)}{\partial t^{\alpha}}=\frac{d}{d t}\left(\frac{1}{\Gamma(1-\alpha)} \int_{0}^{t}(t-r)^{-\alpha} v(r) d r\right)
$$

and $D_{0^{+}}^{\alpha} v(t)=: \frac{d}{d t} v(t)$ if $\alpha=1$. The functions $\psi, H$ in (1.1) are defined later. The operator $\Delta^{*}$ is called Laplace-Beltrami which is introduced in more details in Section 2. To the best of our knowledge, there are not any results on problem (1.1). Our main goal in this paper is to studying two following problem.

- Our first goal is to considering Cauchy initial problem (1.1)-(1.2) under the assumption $\varphi \in \mathrm{H}^{\sigma}\left(\mathrm{S}^{2}\right)$.

- Our second goal is to considering Cauchy terminal problem (1.1)-(1.2) under the assumption $\theta \in$ $H^{\mu}\left(S^{2}\right)$.

This article is organized as follows. Section 2 gives some preliminary and mild solution. In Section 3 , we present our main results including two main theorems. Finally, the proof of some theorems is completed in Section 4.

\section{Preliminaries}

Consider the Mittag-Leffler function, which is defined by

$$
\mathrm{E}_{\alpha, \beta}(\xi)=\sum_{n=1}^{\infty} \frac{\xi^{n}}{\Gamma(n \alpha+\beta)}
$$

$(\xi \in \mathbb{C})$, for $\alpha>0$ and $\beta \in \mathbb{R}$. We call to mind the following lemmas (see for example [22]). We have the following lemma which is useful for next proof.

Lemma 2.1. Let $0<\alpha<1$. Then the function $z \mapsto \mathrm{E}_{\alpha, \alpha}(z)$ has no negative root. Moreover, there exists a constant $\overline{\mathrm{C}}_{\alpha}$ such that

$$
0 \leqslant \mathrm{E}_{\alpha, \alpha}(-z) \leqslant \frac{\overline{\mathrm{C}}_{\alpha}}{1+z}, \quad z>0
$$

Spherical harmonics are polynomials which satisfy $\Delta_{\chi} Y(x)=0$ (where $\Delta_{x}$ is the Laplacian operator in 
$R^{3}$ ) and are restricted to the surface of the Euclidean sphere $S^{2}$. The eigenvalues for $-\Delta$ in $R^{3}$ are

$$
\lambda_{l}=l^{2}+l, l=0,1,2, \ldots
$$

and the eigenfunctions corresponding to $\lambda_{l}$ are the spherical harmonics $\mathbf{Z}_{l}(x)$ of order l, i.e.,

$$
\Delta \mathbf{Z}_{\mathrm{l}}(x)=-\lambda_{\mathrm{l}} \mathbf{Z}_{\mathrm{l}}(x)
$$

The space of all spherical harmonics of degree $l$ on $S^{2}$, denoted by $V_{l}$, has an orthonormal basis $\left\{\mathbf{Z}_{l k}(x)\right.$ : $k=1,2,3, \ldots, N(2, l)\}$, where

$$
\mathrm{N}(2,0)=1, \quad \mathrm{~N}(2, l)=\frac{2 l+1}{\Gamma(2)}, \quad l \geqslant 1 .
$$

Let any function $\psi \in \mathrm{L}^{2}\left(\mathrm{~S}^{n}\right)$, so it is expressed by the expansion of spherical harmonics

$$
\psi=\sum_{l=0}^{\infty} \sum_{k=1}^{\frac{2 l+1}{\Gamma(2)}} \widehat{\psi}_{l k} \mathbf{Z}_{l k}, \widehat{\psi}_{l k}=\int_{S^{2}} \psi \overline{\mathbf{Z}}_{l k} \mathrm{~d} S
$$

where $d S$ is the surface measure of the unit sphere. The Sobolev space $\mathrm{H}^{\sigma}\left(\mathrm{S}^{2}\right)$ is defined by

$$
H^{\sigma}\left(S^{2}\right)=\left\{\psi \in \mathrm{L}^{2}\left(S^{2}\right): \sum_{l=0}^{\infty} \sum_{k=1}^{\frac{2 l+1}{\Gamma(2)}}\left(l^{2}+l+1\right)^{\sigma}\left|\widehat{\psi}_{l k}\right|^{2}<\infty\right\}
$$

with the following norm

$$
\|\psi\|_{H^{\sigma}\left(S^{2}\right)}^{2}=\sum_{l=0}^{\infty} \sum_{k=1}^{\frac{2 l+1}{\Gamma(2)}}\left(l^{2}+l+1\right)^{\sigma}\left|\widehat{\psi}_{l k}\right|^{2}
$$

\section{Main results}

Theorem 3.1. Let $\beta>1 / 2$. Let assume that $\psi:(0, T) \rightarrow \mathbb{R}$ such that

$$
|\psi(z)| \leqslant z^{\delta}, \quad-\frac{1}{2}<\delta<1
$$

Let $\mathrm{u}$ be the solution of Problem (1.1) with the Cauchy initial condition

$$
u(x, 0)=\varphi(x), \quad x \in S^{2} .
$$

Let $\varphi \in \mathrm{H}^{\sigma}\left(\mathrm{S}^{2}\right)$. Then we get $\mathrm{u} \in \mathrm{L}^{\mathrm{q}}\left(0, \mathrm{~T} ; \mathrm{H}^{\sigma}\left(\mathrm{S}^{2}\right)\right)$. Here $\mathrm{q}$ satisfies that

$$
q>\max \left(\frac{1}{1-\beta}, \frac{1}{\frac{1}{2}+\beta \theta-\beta-\delta}\right) .
$$

And $\theta$ is a real number satisfying

$$
\frac{\beta+\delta-3 / 2}{\beta}<\theta<\frac{\beta-1 / 2}{\beta} .
$$

Theorem 3.2. Let $u$ be the solution of Problem (1.1) with the Cauchy initial condition

$$
u(x, T)=\theta(x), \quad x \in S^{2} .
$$

Let $\theta \in \mathrm{H}^{\mu}\left(S^{2}\right)$ and $\mathrm{H} \in \mathrm{L}^{2}\left(0, \mathrm{~T} ; \mathrm{H}^{\mu}\left(S^{2}\right)\right)$. Then we get $\mathrm{u} \in \mathrm{L}^{\mathrm{q}}\left(0, \mathrm{~T} ; \mathrm{H}^{\mu}\left(\mathrm{S}^{2}\right)\right)$. 


\section{Proof of Theorem (3.1)}

Proof. We know from [11] that $\Delta^{*}$ is the Laplace-Beltrami on the sphere $S^{n}$. Any function $u \in \mathrm{L}^{2}\left(\mathrm{~S}^{\mathrm{n}}\right)$ can be described by the terms of spherical harmonics

$$
u(x, t)=\sum_{l=0}^{\infty} \sum_{k=1}^{\frac{2 l+1}{\Gamma(2)}} \widehat{u}_{l k}(t) \mathbf{Z}_{l k}(x), \widehat{u}_{l k}(t)=\int_{S^{n}} u(x, t) \bar{Z}_{l k}(x) d S,
$$

where $\mathrm{dS}$ is the surface measure of the unit sphere. Let us first give an expression of the mild solution. By a simple calculation, we give that

$$
\left(1+a \lambda_{l}\right) \frac{d^{\beta}}{d t^{\beta}} \widehat{u}_{l k}(t)+\lambda_{l} \widehat{u}_{l k}(t)=\widehat{H}_{l k}(t),
$$

where we denote

$$
\widehat{H}_{l k}(s)=\int_{S^{n}} H(x, s) \bar{Y}_{l k}(x) d S .
$$

Therefore, we find that

$$
\frac{d^{\beta}}{d t^{\beta}} \widehat{u}_{l k}(t)+\frac{l^{2}+l}{1+a l^{2}+a l} \widehat{u}_{l k}(t)=\frac{\widehat{H}_{l k}(t)}{1+a l^{2}+a l} .
$$

Let $\varphi=\left.\mathrm{t}^{1-\alpha} \mathfrak{u}\right|_{t=0}$. Then we obtain

$$
\begin{aligned}
\widehat{\mathrm{u}}_{l k}(\mathrm{t})= & \Gamma(\beta) \mathrm{t}^{\beta-1} \mathrm{E}_{\beta, \beta}\left(-\frac{\mathrm{l}^{2}+\mathrm{l}}{1+\mathrm{al} \mathrm{l}^{2}+\mathrm{al}} \mathrm{t}^{\beta}\right) \widehat{\varphi}_{l k} \\
& +\int_{0}^{\mathrm{t}} \frac{1}{1+a l^{2}+\mathrm{al}}(\mathrm{t}-z)^{\beta-1} \mathrm{E}_{\beta, \beta}\left(-\frac{l^{2}+\mathrm{l}}{1+a l^{2}+a l}(\mathrm{t}-z)^{\beta}\right) \widehat{\mathrm{H}}_{l k}(z) \mathrm{d} z .
\end{aligned}
$$

So, we get that

$$
\begin{aligned}
u(x, t)= & \sum_{l=0}^{\infty} \sum_{k=1}^{\frac{2 l+1}{\Gamma(2)}}\left(\Gamma(\alpha) t^{\beta-1} \mathrm{E}_{\beta, \beta}\left(-\frac{l^{2}+l}{1+a l^{2}+a l} t^{\beta}\right) \widehat{\varphi}_{l k}\right) \mathbf{z}_{l k}(x) \\
& +\sum_{l=0}^{\infty} \sum_{k=1}^{\frac{2 l+1}{\Gamma(2)}}\left(\int_{0}^{t} \frac{1}{1+a l^{2}+a l}(t-z)^{\beta-1} \mathrm{E}_{\beta, \beta}\left(-\frac{l^{2}+l}{1+a l^{2}+a l}(t-z)^{\beta}\right) \psi(z) \widehat{H}_{l k}(z) d z\right) \mathbf{z}_{l k}(x) \\
= & D_{1, \beta}(x, t)+D_{2, \beta}(x, t) .
\end{aligned}
$$

The first term is bounded by

$$
\begin{aligned}
\left\|D_{1}(., t)\right\|_{H^{\sigma}\left(S^{2}\right)}^{2} & =\sum_{l=0}^{\infty} \sum_{k=1}^{\frac{2 l+1}{\Gamma(2)}}\left(l^{2}+l+1\right)^{\sigma}\left(\Gamma(\beta) t^{\beta-1} E_{\beta, \beta}\left(-\frac{l^{2}+l}{1+a l^{2}+a l} t^{\alpha}\right) \widehat{\varphi}_{l k}\right)^{2} \\
& \leqslant C_{2, \beta}^{2}|\Gamma(\beta)|^{2} t^{2 \beta-2} \sum_{l=0}^{\infty} \sum_{k=1}^{\frac{2 l+1}{\Gamma(2)}}\left(l^{2}+l+1\right)^{\sigma}\left|\widehat{\varphi}_{l k}\right|^{2} \\
& =C_{2, \beta}^{2}|\Gamma(\beta)|^{2} t^{2 \beta-2}\|\varphi\|_{H^{\sigma}\left(S^{2}\right)}^{2} .
\end{aligned}
$$

Hence, we arrive at

$$
\left\|D_{1}(., t)\right\|_{H^{\sigma}\left(S^{2}\right)} \leqslant C_{2, \beta} \Gamma(\beta) t^{\beta-1}\|\varphi\|_{H^{\sigma}\left(S^{2}\right)} .
$$


It is obvious to see that for any $0<\theta<1$ and $l \geqslant 1$,

$$
\begin{gathered}
E_{\beta, \beta}\left(-\frac{l^{2}+l}{1+a l^{2}+a l} t^{\beta}\right) \leqslant \frac{C_{2, \beta}}{1+\frac{l^{2}+l}{1+a l^{2}+a l} t^{\beta}} \leqslant \frac{C_{2, \beta}}{\left(1+\frac{l^{2}+l}{1+a l^{2}+a l} t^{\beta}\right)^{\theta}} \\
\leqslant C_{2, \beta}\left(\frac{1+a l^{2}+a l}{l^{2}+l}\right)^{\theta} t^{-\beta \theta} \leqslant C_{2, \beta}\left(\frac{1}{2}+a\right)^{\theta} t^{-\beta \theta}, \\
\left\|D_{2}(., t)\right\|_{H^{\sigma}\left(S^{2}\right)}^{2} \\
=\sum_{l=0}^{\infty} \sum_{k=1}^{\frac{2 l+1}{\Gamma(2)}}\left(l^{2}+l+1\right)^{\sigma}\left(\int_{0}^{t} \frac{1}{1+a l^{2}+a l}(t-z)^{\beta-1} E_{\beta, \beta}\left(-\frac{l^{2}+l}{1+a l^{2}+a l}(t-z)^{\beta}\right) \psi(z) \widehat{H}_{l k}(z) d z\right)^{2} \\
\leqslant\left(C_{2, \beta}\left(\frac{1}{2}+a\right)^{\theta}\right)^{2} \sum_{l=0}^{\infty} \sum_{k=1}^{\frac{2 l+1}{\Gamma(2)}}\left(l^{2}+l+1\right)^{\sigma}\left(\int_{0}^{t}(t-z)^{\beta-1-\beta \theta} \psi(z) \widehat{H}_{l k}(z) d z\right)^{2} .
\end{gathered}
$$

Hölder inequality implies immediately that

$$
\left(\int_{0}^{t}(t-z)^{\beta-1-\beta \theta} \psi(z) \widehat{H}_{l k}(z) d z\right)^{2} \leqslant\left(\int_{0}^{t}(t-z)^{2 \beta-2-2 \beta \theta}|\psi(z)|^{2} d z\right)\left(\int_{0}^{t}\left|\widehat{H}_{l k}(z)\right|^{2} d z\right) .
$$

Let us assume that $|\psi(z)|^{2} \leqslant z^{2 \delta}$. Then we get

$$
\int_{0}^{t}(t-z)^{2 \beta-2-2 \beta \theta}|\psi(z)|^{2} d z \leqslant \int_{0}^{t}(t-z)^{2 \beta-2-2 \beta \theta} z^{2 \delta} d z=t^{2 \beta-1-2 \beta \theta+2 \delta} \mathbf{B}(2 \beta-1-2 \beta \theta, 2 \delta+1) .
$$

Here we notice that the condition $0<\theta<\frac{\beta-1 / 2}{\beta}$, which allows us to get that

$$
2 \beta-1-2 \beta \theta>0 \text {. }
$$

This implies that

$$
\begin{aligned}
& \sum_{l=0}^{\infty} \sum_{k=1}^{\frac{2 l+1}{\Gamma(2)}}\left(l^{2}+l+1\right)^{\sigma}\left(\int_{0}^{t}(t-z)^{\beta-1-\beta \theta} \psi(z) \widehat{H}_{l k}(z) d z\right)^{2} \\
& \quad \leqslant t^{2 \beta-1-2 \beta \theta+2 \delta} \mathbf{B}(2 \beta-1-2 \beta \theta, 2 \delta+1) \int_{0}^{t}\left(\sum_{l=0}^{\infty} \sum_{k=1}^{\frac{2 l+1}{\Gamma(2)}}\left(l^{2}+l+1\right)^{\sigma}\left|\widehat{H}_{l k}(z)\right|^{2}\right) d z \\
& \quad=\mathrm{t}^{2 \beta-1-2 \beta \theta+2 \delta} \mathbf{B}(2 \beta-1-2 \beta \theta, 2 \delta+1) \int_{0}^{\mathrm{t}}\|\mathrm{H}(., z)\|_{\mathbf{H}^{\sigma}\left(S^{2}\right)}^{2} \mathrm{~d} z \\
& \leqslant \mathrm{t}^{2 \beta-1-2 \beta \theta+2 \delta} \mathbf{B}(2 \beta-1-2 \beta \theta, 2 \delta+1)\|\mathrm{H}\|_{\mathrm{L}^{2}\left(0, \mathrm{~T} ; \mathbf{H}^{\sigma}\left(\mathrm{S}^{2}\right)\right)}^{2} .
\end{aligned}
$$

So, it follows from (4.5) that

$$
\left\|\mathrm{D}_{2}(., \mathrm{t})\right\|_{\mathbf{H}^{\sigma}\left(\mathrm{S}^{2}\right)}^{2} \leqslant\left(\mathrm{C}_{2, \beta}\left(\frac{1}{2}+\mathrm{a}\right)^{\theta}\right)^{2} \mathbf{B}(2 \beta-1-2 \beta \theta, 2 \delta+1) \mathrm{t}^{2 \beta-1-2 \beta \theta+2 \delta}\|\mathrm{H}\|_{\mathrm{L}^{2}\left(0, \mathrm{~T} ; \mathbf{H}^{\sigma}\left(\mathrm{S}^{2}\right)\right)}^{2} .
$$

By combining (4.2), (4.3), (4.6), we find that

$$
\begin{aligned}
\|u(., t)\|_{H^{\sigma}\left(S^{2}\right)} & \leqslant\left\|D_{1}(., t)\right\|_{H^{\sigma}\left(S^{2}\right)}+\left\|D_{2}(., t)\right\|_{H^{\sigma}\left(S^{2}\right)} \\
& \leqslant C_{2, \beta} \Gamma(\beta) t^{\beta-1}\|\varphi\|_{H^{\sigma}\left(S^{2}\right)}+M(\beta, \theta, \delta) t^{\beta-1 / 2-\beta \theta+\delta}\|H\|_{L^{2}\left(0, T ; H^{\sigma}\left(S^{2}\right)\right)^{\prime}}
\end{aligned}
$$


where we denote

$$
M(\beta, \theta, \delta)=C_{2, \beta}\left(\frac{1}{2}+a\right)^{\theta} \sqrt{\mathbf{B}(2 \beta-1-2 \beta \theta, 2 \delta+1)} .
$$

It follows from (4.7) that

$$
\begin{aligned}
\|u\|_{L^{q}\left(0, T ; H^{\sigma}\left(S^{2}\right)\right)} \leqslant & C_{2, \beta} \Gamma(\beta)\|\varphi\|_{H^{\sigma}\left(S^{2}\right)}\left(\int_{0}^{T} t^{(\beta-1) \mathrm{q}} d t\right) \\
& +M(\beta, \theta, \delta)\|H\|_{L^{2}\left(0, T ; H^{\sigma}\left(S^{2}\right)\right)}\left(\int_{0}^{T} t^{(\beta-1 / 2-\beta \theta+\delta) q} d t\right) .
\end{aligned}
$$

By conditions (3.1) and (3.2), we know that

$$
(\beta-1) q+1>0, \quad(\beta-1 / 2-\beta \theta+\delta) q+1>0 .
$$

Therefore, the proper integrals $\int_{0}^{T} t^{(\beta-1) q} d t$ and $\int_{0}^{T} t^{(\beta-1 / 2-\beta \theta+\delta) q} d t$ are convergent. This conclude that $u \in \mathrm{L}^{\mathrm{q}}\left(0, \mathrm{~T} ; \mathbf{H}^{\mathrm{\sigma}}\left(\mathrm{S}^{2}\right)\right)$.

\subsection{Proof of Theorem (3.2)}

From the fomula (4.1), we let $t=T$ to get that

$$
\begin{aligned}
\widehat{\theta}_{l k}=\widehat{u}_{l k}(T)= & \Gamma(\beta) T^{\beta-1} E_{\beta, \beta}\left(-\frac{l^{2}+l}{1+a l^{2}+a l} T^{\beta}\right) \widehat{\varphi}_{l k} \\
& +\int_{0}^{T} \frac{(T-z)^{\beta-1}}{1+a l^{2}+a l} E_{\beta, \beta}\left(-\frac{l^{2}+l}{1+a l^{2}+a l}(T-z)^{\beta}\right) \psi(z) \widehat{H}_{l k}(z) d z .
\end{aligned}
$$

Hence, we find that

$$
\widehat{\varphi}_{l k}=\frac{\widehat{\theta}_{l k}-\int_{0}^{T} \frac{(T-z)^{\beta-1}}{1+a l^{2}+a l} E_{\beta, \beta}\left(-\frac{l^{2}+l}{1+a l^{2}+a l}(T-z)^{\beta}\right) \psi(z) \widehat{H}_{l k}(z) d z}{\Gamma(\beta) T^{\beta-1} E_{\beta, \beta}\left(-\frac{l^{2}+l}{1+a l^{2}+a l} T^{\beta}\right)} .
$$

Hence, we find that

$$
\begin{aligned}
& \widehat{u}_{l k}(t)=\frac{t^{\beta-1} E_{\beta, \beta}\left(-\frac{l^{2}+l}{1+a l^{2}+a l} t^{\beta}\right)}{T^{\beta-1} E_{\beta, \beta}\left(-\frac{l^{2}+l}{1+a l^{2}+a l} T^{\beta}\right)} \widehat{\theta}_{l k} \\
& -\frac{t^{\beta-1} E_{\beta, \beta}\left(-\frac{l^{2}+l}{1+a l^{2}+a l} t^{\beta}\right)}{T^{\beta-1} E_{\beta, \beta}\left(-\frac{l^{2}+l}{1+a l^{2}+a l} T^{\beta}\right)}\left(\int_{0}^{T} \frac{(T-z)^{\beta-1}}{1+a l^{2}+a l} E_{\beta, \beta}\left(-\frac{l^{2}+l}{1+a l^{2}+a l}(T-z)^{\beta}\right) \psi(z) \widehat{H}_{l k}(z) d z\right) \\
& +\int_{0}^{t} \frac{1}{1+a l^{2}+a l}(t-z)^{\beta-1} E_{\beta, \beta}\left(-\frac{l^{2}+l}{1+a l^{2}+a l}(t-z)^{\beta}\right) \psi(z) \widehat{H}_{l k}(z) d z .
\end{aligned}
$$

So, we get immediately that

$$
\begin{aligned}
u(x, t)= & \sum_{l=0}^{\infty} \sum_{k=1}^{\frac{2 l+1}{\Gamma(2)}}\left(\frac{t^{\beta-1} E_{\beta, \beta}\left(-\frac{l^{2}+l}{1+a l^{2}+a l} t^{\beta}\right)}{T^{\beta-1} E_{\beta, \beta}\left(-\frac{l^{2}+l}{1+a l^{2}+a l} T^{\beta}\right)} \widehat{\psi}_{l k}\right) \mathbf{z}_{l k}(x)-\sum_{l=0}^{\infty} \sum_{k=1}^{\frac{2 l+1}{\Gamma(2)}}\left(\frac{t^{\beta-1} E_{\beta, \beta}\left(-\frac{l^{2}+l}{1+a l^{2}+a l} t^{\beta}\right)}{T^{\beta-1} E_{\beta, \beta}\left(-\frac{l^{2}+l}{1+a l^{2}+a l} T^{\beta}\right)}\right. \\
& \left.\times\left(\int_{0}^{T} \frac{(T-z)^{\beta-1}}{1+a l^{2}+a l} E_{\beta, \beta}\left(-\frac{l^{2}+l}{1+a l^{2}+a l}(T-z)^{\beta}\right) \psi(z) \widehat{H}_{l k}(z) d z\right)\right)
\end{aligned}
$$




$$
\begin{aligned}
& \times \mathbf{Z}_{l k}(x)+\sum_{l=0}^{\infty} \sum_{k=1}^{\frac{2 l+1}{\Gamma(2)}}\left(\int_{0}^{t} \frac{1}{1+a l^{2}+a l}(t-z)^{\beta-1} E_{\beta, \beta}\left(-\frac{l^{2}+l}{1+a l^{2}+a l}(t-z)^{\beta}\right) \psi(z) \widehat{H}_{l k}(z) d z\right) Z_{l k}(x) \\
= & G_{1}(x, t)+G_{2}(x, t)+G_{3}(x, t) .
\end{aligned}
$$

Due to the upper and lower estimates of Mittag-Lefler function $E_{\alpha, \alpha}$, we note that

$$
\frac{t^{\beta-1} E_{\beta, \beta}\left(-\frac{l^{2}+l}{1+a l^{2}+a l} t^{\beta}\right)}{T^{\beta-1} E_{\beta, \beta}\left(-\frac{l^{2}+l}{1+a l^{2}+a l} T^{\beta}\right)} \leqslant T^{1-\beta} t^{\beta-1} \frac{\frac{C_{2, \beta}}{1+\frac{l^{2}+l}{1+a l^{2}+a l} t^{\beta}}}{\frac{C_{1, \beta}}{1+\frac{l^{2} l}{1+a l^{2}+a l} T^{\beta}}} \leqslant \frac{T^{1-\beta} C_{2, \beta}}{C_{1, \beta}}\left(1+\frac{T^{\beta}}{a}\right) t^{\beta-1}=C_{3, \beta} t^{\beta-1},
$$

where we denote

$$
C_{3, \beta}=\frac{T^{1-\beta} C_{2, \beta}}{C_{1, \beta}}\left(1+\frac{T^{\beta}}{a}\right) .
$$

For the first term of the right hand side of (4.8), we get that

$$
\begin{aligned}
\left\|G_{1}(., t)\right\|_{H^{\mu}\left(S^{2}\right)}^{2} & =\sum_{l=0}^{\infty} \sum_{k=1}^{\frac{2 l+1}{\Gamma(2)}}\left(l^{2}+l+1\right)^{\mu}\left(\frac{t^{\beta-1} E_{\beta, \beta}\left(-\frac{l^{2}+l}{1+a l^{2}+a l} t^{\beta}\right)}{T^{\beta-1} E_{\beta, \beta}\left(-\frac{l^{2}+l}{1+a l^{2}+a l} T^{\beta}\right)} \widehat{\theta}_{l k}\right)^{2} \\
& \leqslant\left|C_{3, \beta} t^{\beta-1}\right|^{2} \sum_{l=0}^{\infty} \sum_{k=1}^{\frac{2 l+1}{\Gamma(2)}}\left(l^{2}+l+1\right)^{\mu}\left|\widehat{\theta}_{l k}\right|^{2}=\left|C_{3, \beta} t^{\beta-1}\right|^{2}\|\theta\|_{H^{\mu}\left(S^{2}\right)}^{2} .
\end{aligned}
$$

Hence, we find that

$$
\left\|G_{1}(., t)\right\|_{H^{\mu}\left(S^{2}\right)} \leqslant C_{3, \beta}\|\theta\|_{H^{\mu}\left(S^{2}\right)} t^{\beta-1} .
$$

For the second term of the right hand side of (4.8) and using (4.4), we get that

$$
\begin{aligned}
& \left\|G_{2}(., t)\right\|_{H^{\mu}\left(S^{2}\right)}^{2}=\sum_{l=0}^{\infty} \sum_{k=1}^{\frac{2 l+1}{\Gamma(2)}}\left(\frac{t^{\beta-1} E_{\beta, \beta}\left(-\frac{l^{2}+l}{1+a l^{2}+a l} t^{\beta}\right)}{T^{\beta-1} E_{\beta, \beta}\left(-\frac{l^{2}+l}{1+a l^{2}+a l} T^{\beta}\right)}\right)^{2} \\
& \times\left(\int_{0}^{T} \frac{(T-z)^{\beta-1}}{1+a l^{2}+a l} E_{\beta, \beta}\left(-\frac{l^{2}+l}{1+a l^{2}+a l}(T-z)^{\beta}\right) \psi(z) \widehat{H}_{l k}(z) d z\right)^{2} \\
& \leqslant\left|C_{3, \beta} t^{\beta-1}\right|^{2} \sum_{l=0}^{\infty} \sum_{k=1}^{\frac{2 l+1}{\Gamma(2)}}\left(l^{2}+l+1\right)^{\mu} \\
& \times\left(\int_{0}^{T} \frac{1}{1+a l^{2}+a l}(T-z)^{\beta-1} E_{\beta, \beta}\left(-\frac{l^{2}+l}{1+a l^{2}+a l}(T-z)^{\beta}\right) \psi(z) \widehat{H}_{l k}(z) d z\right)^{2} \\
& \leqslant\left|C_{3, \beta} t^{\beta-1}\right|^{2}\left(C_{2, \beta}\left(\frac{1}{2}+a\right)^{\theta}\right)^{2} \sum_{l=0}^{\infty} \sum_{k=1}^{\frac{2 l+1}{\Gamma(2)}}\left(l^{2}+l+1\right)^{\mu}\left(\int_{0}^{T}(T-z)^{\beta-1-\beta \theta} \psi(z) \widehat{H}_{l k}(z) d z\right)^{2} \\
& \leqslant\left|C_{3, \beta} t^{\beta-1}\right|^{2}\left(C_{2, \beta}\left(\frac{1}{2}+a\right)^{\theta}\right)^{2} \\
& \times \sum_{l=0}^{\infty} \sum_{k=1}^{\frac{2 l+1}{\Gamma(2)}}\left(l^{2}+l+1\right)^{\mu}\left(\int_{0}^{T}(T-z)^{2 \beta-2-2 \beta \theta}|\psi(z)|^{2} \mathrm{~d} z\right)\left(\int_{0}^{T}\left|\widehat{H}_{l k}(z)\right|^{2} \mathrm{~d} z\right) .
\end{aligned}
$$


Since the fact that $|\psi(z)| \leqslant z^{\delta}$, we find that

$$
\int_{0}^{T}(T-z)^{2 \beta-2-2 \beta \theta}|\psi(z)|^{2} d z \leqslant \int_{0}^{T}(T-z)^{2 \beta-2-2 \beta \theta} z^{2 \delta} d z=T^{2 \beta-1-2 \beta \theta+2 \delta} \mathbf{B}(2 \beta-1-2 \beta \theta, 2 \delta+1) .
$$

From two above observations, we arrive at

$$
\begin{aligned}
\left\|G_{2}(., t)\right\|_{H^{\mu}\left(S^{2}\right)}^{2} \leqslant & \left|C_{3, \beta} t^{\beta-1}\right|^{2}\left(C_{2, \beta}\left(\frac{1}{2}+a\right)^{\theta}\right)^{2} T^{2 \beta-1-2 \beta \theta+2 \delta} \mathbf{B}(2 \beta-1-2 \beta \theta, 2 \delta+1) \\
& \times\left(\int_{0}^{T}\left(\sum_{l=0}^{\infty} \sum_{k=1}^{\frac{2 l+1}{\Gamma(2)}}\left(l^{2}+l+1\right)^{\mu}\left|\widehat{H}_{l k}(z)\right|^{2}\right) d z\right) \\
\leqslant & \left|C_{3, \beta} t^{\beta-1}\right|^{2}\left(C_{2, \beta}\left(\frac{1}{2}+a\right)^{\theta}\right)^{2} T^{2 \beta-1-2 \beta \theta+2 \delta} \mathbf{B}(2 \beta-1-2 \beta \theta, 2 \delta+1)\|H\|_{L^{2}\left(0, \mathrm{~T} ; \mathbf{H}^{\mu}\left(S^{2}\right)\right)}^{2} .
\end{aligned}
$$

Hence, we obtain that

$$
\left\|G_{2}(., t)\right\|_{H^{\mu}\left(S^{2}\right)} \leqslant C_{4, \beta, \theta, \delta}\|H\|_{L^{2}\left(0, T ; H^{\mu}\left(S^{2}\right)\right)} t^{\beta-1},
$$

where

$$
C_{4, \beta, \theta, \delta}=C_{3, \beta} C_{2, \beta}\left(\frac{1}{2}+a\right)^{\theta} \sqrt{T^{2 \beta-1-2 \beta \theta+2 \delta} \mathbf{B}(2 \beta-1-2 \beta \theta, 2 \delta+1)} .
$$

By using a similar argument as in (4.6), we find that

$$
\left\|G_{3}(., t)\right\|_{H^{\mu}\left(S^{2}\right)} \leqslant M(\beta, \theta, \delta) t^{\beta-1 / 2-\beta \theta+\delta}\|H\|_{L^{2}\left(0, T ; H^{\mu}\left(S^{2}\right)\right)} .
$$

Combining (4.9), (4.10), (4.11), we arrive at

$$
\begin{aligned}
\|u(., t)\|_{H^{\mu}\left(S^{2}\right) \leqslant} & \left\|G_{1}(., t)\right\|_{H^{\mu}\left(S^{2}\right)}+G_{2}(., t)\left\|_{H^{\mu}\left(S^{2}\right)}+\right\| G_{3}(., t) \|_{H^{\mu}\left(S^{2}\right)} \\
\leqslant & C_{3, \beta}\|\theta\|_{H^{\mu}\left(S^{2}\right)} t^{\beta-1}+C_{4, \beta, \theta, \delta}\|H\|_{L^{2}\left(0, T ; H^{\mu}\left(S^{2}\right)\right)} t^{\beta-1} \\
& +M(\beta, \theta, \delta) t^{\beta-1 / 2-\beta \theta+\delta}\|H\|_{L^{2}\left(0, T ; H^{\mu}\left(S^{2}\right)\right)} .
\end{aligned}
$$

\section{References}

[1] K. A. Abro, A. Atangana, Mathematical analysis of memristor through fractal-fractional differential operators: a numerical study, Math. Methods Appl. Sci., 43 (2020), 6378-6395. 1

[2] A. Atangana, A. Akgül, K. M. Owolabi, Analysis of fractal fractional differential equations, Alexandria Eng. J., 59 (2020), 1117-1134.

[3] A. Atangana, E. Bonyah, Fractional stochastic modeling: New approach to capture more heterogeneity, Chaos, 29 (2019), 13 pages. 1

[4] A. Atangana, E. F. D. Goufo, Cauchy problems with fractal-fractional operators and applications to groundwater dynamics, Fractals, 28 (2020), 21 pages.

[5] A. Atangana, Z. Hammouch Fractional calculus with power law: The cradle of our ancestors, Eur. Phys. J. Plus, 134 (2019), 13 pages. 1

[6] Z. Brzeźniak, B. Goldys, Q. T. Le Gia, Random attractors for the stochastic Navier-Stokes equations on the 2D unit sphere, J. Math. Fluid Mech., 20 (2018), 227-253. 1

[7] R. M. Ganji, H. Jafari, S. Nemati, A new approach for solving integro-differential equations of variable order, J. Comput. Appl. Math., 379 (2020), 13 pages. 1

[8] H. Jafari, H. Tajadodi, R. M. Ganji, A numerical approach for solving variable order differential equations based on Bernstein polynomials, Comput. Math. Methods, 1 (2019), 11 pages. 1

[9] S. Kumar, A. Atangana, A numerical study of the nonlinear fractional mathematical model of tumor cells in presence of chemotherapeutic treatment, Int. J. Biomath., 13 (2020), 17 pages. 1

[10] Q. T. Le Gia, Galerkin approximation of elliptic PDEs on spheres, J. Approx. Theory, 130 (2004), 125-149. 1 
[11] Q. T. Le Gia, Approximation of parabolic PDEs on spheres using collocation method, Adv. Comput. Math., 22 (2005), 377-397. 1, 4

[12] Q. T. Le Gia, I. H. Sloan, T. Tran, Overlapping additive Schwarz preconditioners for elliptic PDEs on the unit sphere, Math. Comp., 78 (2009), 79-101. 1

[13] Q. T. Le Gia, N. H. Tuan, T. Tran, Solving the backward heat equation on the unit sphere, ANZIAM J. Electron. Suppl., 56 (2014), 262-278. 1

[14] N. H. Luc, H. Jafari, P. Kumam, N. H. Tuan, On an initial value problem for time fractional pseudo-parabolic equation with Caputo derivarive, Mathematical Methods in the Applied Sciences, (2021), to appear. 1

[15] J. Manimaran, L. Shangerganesh, A. Debbouche, A time-fractional competition ecological model with cross-diffusion, Math. Methods Appl. Sci., 43 (2020), 5197-5211.

[16] J. Manimaran, L. Shangerganesh, A. Debbouche, Finite element error analysis of a time-fractional nonlocal diffusion equation with the Dirichlet energy, J. Comput. Appl. Math., 382 (2021), 11 pages.

[17] T. B. Ngoc, T. Caraballo, N. H. Tuan, Y. Zhou, Existence and regularity results for terminal value problem for nonlinear super-diffusive fractional wave equations, Nonlinearity, 34 (2021), 12 pages.

[18] T. B. Ngoc, Y. Zhou, D. O'Regan, N. H. Tuan, On a terminal value problem for pseudoparabolic equations involving Riemann-Liouville fractional derivatives, Appl. Math. Lett., 106 (2020), 9 pages.

[19] O. Nikan, H. Jafari, A. Golbabai, Numerical analysis of the fractional evolution model for heat flow in materials with memory, Alexandria Eng. J., 59 (2020), 2627-2637. 1

[20] C. V. Pao, Reaction diffusion equations with nonlocal boundary and nonlocal initial conditions, J. Math. Anal. Appl., 195 (1995), 702-718.

[21] N. D. Phuong, N. H. Luc, Note on a Nonlocal Pseudo-Parabolic Equation on the Unit Sphere, Dyn. Syst. Appl., 30 (2021), 295-304. 1

[22] I. Podlubny, Fractional Differential Equations, Academic Press, San Diego, (1999). 2

[23] M. Ruzhansky, N. Tokmagambetov, B. T. Torebek, On a non-local problem for a multi-term fractional diffusion-wave equation, Fract. Calc. Appl. Anal., 23 (2020), 324-355.

[24] K. Sakamoto, M. Yamamoto, Initial value/boundary value problems for fractional diffusion-wave equations and applications to some inverse problems, J. Math. Anal. Appl., 382 (2011), 426-447.

[25] N. H. Sweilam, S. M. Al-Mekhlafi, T. Assiri, A. Atangana, Optimal control for cancer treatment mathematical model using Atangana-Baleanu-Caputo fractional derivative, Adv. Difference Equ., 2020 (2020), 21 pages. 1

[26] N. H. Tuan, A. Debbouche, T. B. Ngoc, Existence and regularity of final value problems for time fractional wave equations, Comput. Math. Appl., 78 (2019), 1396-1414.

[27] N. H. Tuan, L. N. Huynh, T. B. Ngoc, Y. Zhou, On a backward problem for nonlinear fractional diffusion equations, Appl. Math. Lett., 92 (2019), 76-84. 1

[28] N. H. Tuan, Y. Zhou, T. N. Thach, N. H. Can, Initial inverse problem for the nonlinear fractional Rayleigh-Stokes equation with random discrete data, Commun. Nonlinear Sci. Numer. Simul., 78 (2019), 18 pages. 